\title{
Uveitis as an extraarticular manifestation in spondyloarthritis
}

\begin{tabular}{c} 
Razvan Adrian Ionescu \\
Colentina Clinical Hospital, Bucharest, Romania \\
"Carol Davila" University of Medicine and Pharmacy, Bucharest, Romania \\
\hline
\end{tabular}

ABSTRACT
Spondylatrhritis is a multifaceted disease, primary involving the axial skeleton. But sometimes, the patient pres-
ent either with articular involvement plus other organ invelovement, or with only extraarticular involvement. As
the most frequent, uveitis is the main extraarticular manifestation (EAM) of spondylarthritis. This EAM is very
important because it can be the first manifestation of SpA, so it may bring the patient to the doctor, be it an
ophthalmologist or a rheumatologist. Diagnosing uveitis in the context of SpA, is of paramount importance also
because anterior uveitis is an emergency, because it can lead to blindness. So, knowing the clinical appearance
and the treatment of uveitis in the context of SpA is crucial for providing the best care for SpA patients.
Keywords: spondylarthritis, extraarticular manifestations, uveitis, emergency

\section{INTRODUCTION}

Spondyloarthritis (SpA) are a group of closely related and sometimes overlapping inflammatory rheumatological diseases that share some features, of which inflammation of the axial skeleton, dactylitis (also known as sausage digits), enthesitis (which is inflammation of the insertion points of the ligaments and tendons on the bone), presence of HLA-B27 are the most prominent ones.

Aside from these common characteristics, another rather frequently encountered feature of this group of diseases, is the presence of extraarticular involvement or extra articular manifestations (EAMs) [1].

The mechanism by which EAMs occur in SpA, is, in brief, the hyperstimulation of the immune system, which becomes systemic and involves different conjunctival structures of the body.

On average, around $40 \%$ of patients with $\mathrm{SpA}$ have at least one EMA during the course of their disease. The frequency of EAMs is dependent on the duration of the underlying SpA: the longer the duration, the more frequent the EAMs; for example the prevalence of uveitis doubles in patients with a more than 20 year-long disease versus those with less than 10 year-long disease [2].

The main feature of uveitis (that is inflammation of one or more of the uveal tract components) in "red eye". A red eye signifies inflammation and is due to the dilation of ocular blood vessels; but, in order to make a proper differential, it is important to know what vessels are inflamed since the congestion of the conjunctival posterior vessels produces superficial inflammation (conjunctivitis, blefaritis) and the congestion of the anterior ciliary vessels produces produces profound inflammation (keratitis, uveitis). But not all red eye situations are an emergency. Only when it coexists with pain or decrease in visual acuity, the red eye is an emergency that implies getting very fast to an ophthalmologist for proper assessment and treatment. The emergency in diagnosing and treating uveitis is the possibility that the healing of the inflammation of the uveal tract occurs with defect (consisting of opacities in the uvea), leading to potential blindness. When the red eye is an emergency, it usually denotes the aggravation of the underlying disease (in this case, of the spondylarthritis). Maybe it is worth noting that there are some 
ophthalmologic emergencies, related to rheumatological conditions, in the absence of a red eye, as well. For instance, in juvenile idiopathic arthritis there is a possibility to have anterior chronic uveitis, which is frequently bilateral but with no visible inflammation (that is no pain or any clinically detectable sign in the beginning) and sometimes with onset directly by a complication; and this is why these patients must be regularly evaluated, even if asymptomatic [3].

The ophthalmologist may be the first specialist involved in recognizing that the uveitis is part of a systemic rheumatological disease. In sarcoidosis, ocular symptoms are the most frequent initial manifestations; in giant cell arteritis, $20 \%$ of patients have only ophthalmologic involvement, with no general symptoms; recurrent polycondritis, $18 \%$ of patients have ocular initial symptoms; last, but not least, Behcet's disease has an initial ophthalmic involvement in a third of patients.

\section{CLASSIFICATION AND DIFFERENTIAL DIAGNOSIS}

The classification of the uveitis is dependent on the predominant location of the inflammation: an anterior uveitis is still anterior, even if, as a consequence of its presence, complications occur on the posterior pole of the eye. Anterior uveitis can be acute (with a sudden onset and limited duration), recurrent (with repeated flares, separated by at least 3 months periods of no uveitis without treatment) and chronic (with flares lasting more than 3 months and relapses within 3 months of no treatment).

Anterior acute uveitis (AAU) is usually immune mediated, not infectious in etiology. In different diseases, there are different locations of the AAU and different prevalence (see Table 1).

TABLE 1. Different diseases and different locations of $A A U$

\begin{tabular}{|l|l|}
\hline Autoimmune disease & Most common associated uveitis \\
\hline SpA [4] & Anterior \\
\hline Behçet's disease [5] & Posterior and panuveitis \\
\hline PsA [6,7] & Anterior \\
\hline JIA [8] & Anterior \\
\hline Sarcoidosis [9,10] & Anterior/posterior/panuveitis \\
\hline IBD [11] & Anterior and/or intermediate \\
\hline Psoriasis & Anterior \\
\hline MS [12] & Intermediate \\
\hline $\begin{array}{l}\text { Vogt-Koyanagi-Harada's } \\
\text { syndrome [12] }\end{array}$ & Posterior/panuveitis \\
\hline
\end{tabular}

HLA-B27 associated AAU is variable in presentation, depending on the underlying HLA-B27 associated disease (see Table 2).

TABLE 2. Characteristics of HLA-B27 associated $A A U$

\begin{tabular}{|l|l|}
\hline SpA [13-15] & IBD or PsA [13] \\
\hline Anterior & Anterior and intermediary \\
\hline $\begin{array}{l}\text { Sudden onset, with } \\
\text { prodrome }\end{array}$ & Sudden onset or insidious onset \\
\hline Unilateral & Unilateral or bilateral \\
\hline $\begin{array}{l}\text { Recurrent, frequently on } \\
\text { the same eye }\end{array}$ & Recurrent or chronic \\
\hline $\begin{array}{l}\text { Complete resolution in } \\
\text { months (or less) }\end{array}$ & Involvement of vitrous body \\
\hline Predominant in male & Predominant in women (IBD) \\
\hline
\end{tabular}

Usually, in HLA-B27 positive SpA patients, uveitis is anterior $(90.5 \%$ of cases), acute $(88.7 \%)$, unilateral $(87.3 \%)$, recurrent $(50.6 \%)$ on the same eye (simultaneous involvement is rare), sometimes with a "flip-flop" pattern (alternative involvement of the eyes), with acute onset with ocular pain before the inflammation of the anterior chamber of the eye, which is severe: red painful eye, photophobia, increased lacrimal secretion; sometimes, it is complicated with hypopyon or posterior iris residual fibrin connections and glaucoma. Its intensity is not related to the SpA activity [4,16-18]. The prevalence of uveitis is also dependent on the type of HLA-B27 related disease, with the higher prevalence in ankylosing spondylitis (AS) (around 33\%) and the lowest in inflammatory bowel disease (IBD) [19]. The incidence of AAU in patients with AS is increasing with the duration of the disease, so that it reaches a 20 -year cumulative value of $24.5 \%$ [20]. But, is there any difference in prevalence of AAU between AS and SpA?! In 2016, de Winter et al. showed that in terms of current uveitis, there is no significant difference between the two, but in terms of the presence of uveitis, ever, the ocular involvement is statistically significantly higher in AS than in SpA [21].

The significance of AAU in SpA is that of a negative prognostic marker: it is associated with higher disease activity scores, with decreased mobility and functionality and, most importantly, with a higher degree of structural damage $[22,23]$. So, the importance of AAU in early diagnosis of SpA lies in the facts the AAU is often the first manifestation in aroud $41 \%$ of patients with SpA, between 41 and $77 \%$ of patients with AAU have actual undiagnosed $\mathrm{SpA}$, which is important as the medium time between onset and diagnosis of disease is around 7 years [24-28]. 


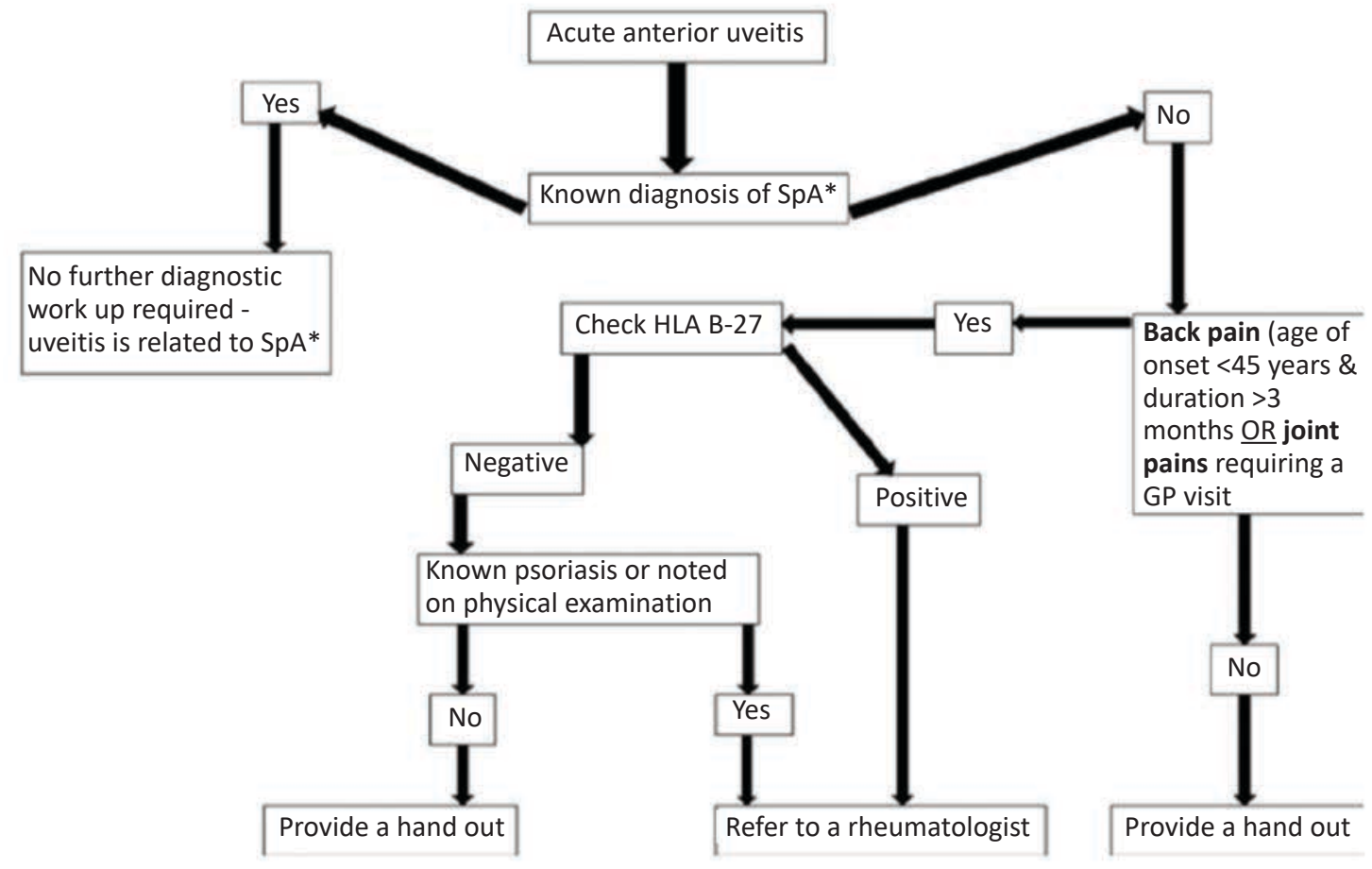

FIGURE 1. The DUET algorithm

\section{MANAGEMENT ALGORITHM}

Such a patient, with acute anterior uveitis that gets to the ophthalmologist, must be sometimes sent to the rheumatologist; in order to ease this process, in 2014, Haroon et al. came up with an evaluation tool which helps ophtalmologists decide what patient with AAU should be sent to the rheumatologist. This is called the Dublin Uveitis Evaluation Tool (DUET) and has a sensibility of $96 \%$ and a specificity of $97 \%$ for deciding when to refer the patient to the rheumatologist (see Figure 1) [29].

Treatment of acute anterior uveitis is, initially, just local with corticosteroids in the form of eye droplets and cycloplegic medicines. This treatment is reserved for easy to moderate cases. When such therapeutic approach does not work, the ophthalmologist will perform periocular cortisone injections and if these fail too, the next step of the therapeutic ap- proach is to use conventional synthetic DMARDs (whose efficacy on AAU is, if ever, quite late) of biologic therapies. When using biologics in the treatment of SpA related AAU, we have to bear in mind that not all biologics have the same efficacy; that is, monoclonal antibodies are preferred over fusion receptor proteins with respect to the incidence of relapses of AAU [30,31].

\section{CONCLUSIONS}

To conclude about uveitis in spondyloarthritis, one might want to remember that AAU may be the first manifestation of $\mathrm{SpA}$, the clinical picture depends on the subset $\mathrm{SpA}$, uveitis may be really severe, when treating it, we prefer monoclonal antibodies over fusion receptors and that the collaboration between ophtalmologists and rheumatologists is crucial for an early diagnosis and an efficacious treatment.

\section{REFERENCES}

1. Mielants $\mathrm{H}$, Van den Bosch F. Extraarticular manifestations. Clin Exp Rheumatol. 2009;27:S56. 
based matched cohort study. Ann Rheum Dis. 2015;74(7):13731378

3. Zeboulon N, Dougados M, Gossec L. Prevalence and characteristics of uveitis in the spondylarthropaties: a systematic literature review. Ann Rheum Dis. 2008;67(7):955-959.

4. Dalvi SR, Yildirim R, Yazici Y. Behcet's syndrome. Drugs. 2012;72:2223-2241.

5. Fraga NA, de Oliveira MF, Follador I, et al. Psoriasis and uveitis: a literature review. An Bras Dermatol. 2012;1270:269-82.

6. Lambert JR, Wright V. Eye inflammation in psoriatic arthritis. Ann Rheum Dis. 1976;35:354-6.

7. Clarke SLN, Sen ES, Ramanan AV. Juvenile idiopathic arthritisassociated uveitis. Pediatr Rheumatol. 2016;14:27.

8. Kobak S. Sarcoidosis: a rheumatologist's perspective. Ther Adv Musculoskelet Dis. 2015;7:196-205.

9. 9Pan J, Kapur M, McCallum R. Noninfectious immune-mediated uveitis and ocular inflammation Curr Allerg Asthma Rep. 2014:14:409.

10. Yanoff M, Cameron D. Diseases of the visual system. In: Goldman L, Schafer Al, eds. Goldman's Cecil Medicine. 24th ed. Philadelphia, PA: Elsevier Saunders; 2012:2426-2442.

11. van Laar JAM, Rothova A, Missotten T, Kuijpers RWAM, van Hagen PM, van Velthoven MEJ. Diagnosis and treatment of uveitis; not restricted to the ophthalmologist. J Clin Transl Res. 2015;1(2):94-99.

12. Rosenbaum JT. Uveitis in spondylarthritis including psoriatic arthritis, ankylosing spondylitis and inflammatory bowel disease. Clin Rheumatol. 2015;34:999-1002.

13. Power WJ, Rodriguex A, Pedroza-Seres M, et al. Outcomes in anterior uveitis associated with the HLA-B27 haplotype. Ophthalmology 1998;105(9):1646-1651.

14. Rosenbaum JT. New developments in uveitis associated with HLA B27. Curr Opin Rheumatol. 2017;29(4):298-303.

15. Khan MA, Haroon M, Rosenbaum JT. Acute anterior uveitis and spondyloarthritis: more than meets the eye. Curr Rheumatol Rep. 2015;17(9):59.

16. an der Linden S, Rentsch HU, Gerber N, et al. The association between ankylosing spondylitis, acute anterior uveitis and HLA-B27: the results of a Swiss family study. Br J Rheumatol. 1988;27(Suppl 2):39-41.

17. Linder R, Hoffman A, Brunner R. Prevalence of the spondylartritides in patients with uveitis. J Rheumatol. 2004;31:2226-2229.

18. Canoui-Poitrine F, Kemta Lekpa F, Farrenq V, et al. Prevalence and factors associated with uveitis in spondylarthritis patients in France: results from an observational survey. Arthritis Care Res. 2012:64:919-924.
19. Stolwijk C, Essers I, van Tubergen A, Boonen A, Bazelier MT, De Bruin ML, de Vries F. The epidemiology of extra-articular manifestations in ankylosing spondylitis: a population-based matched cohort study. Ann Rheum Dis. 2015;74(7):1373-8.

20. De Winter et al. Prevalence of peripheral and extra-articular disease in ankylosing spondylitis versus non-radiographic axial spondyloarthritis: a meta-analysis. Arthritis Res Ther. 2016;18:196.

21. Chen $\mathrm{CH}$, Lin KC, Chen HA, et al. Association of acute anterior uveitis with disease activity, functional ability and physical mobility in patients with ankylosing spondilitis: a cross-sectional study of chinese patients in Taiwan. Clin Rheumatol. 2007;26:953-957.

22. Essers I, Ramiro S, Stolwijk C, et al. Characteristics associated with the presence and development of extraarticular manifestations in ankylosing spondylitis:12 year results from OASIS. Rheumatology (Oxford). 2015;54(4):633-640.

23. Poddubnyy $D$, van Tubergen $A$, Landewe $R$, et al. Develpomenet of an ASAS-endorsed recommendation for the early referral of patients with a suspicion of axial spondylarthritis. Ann Rheum Dis. 2015;74:1483-1487.

24. Feldtkeller E, Bruckel J, Kahn MA. Scientific contributions of ankylosing spondylitis patient advocacy groups. Curr Opin Rheumatol. 2000:12:239-47.

25. Pato E, Banares A, Jover JA, et al. Undiagnosed spondylarthropathy in patients presenting with anterior uveitis. $J$ Rheumatol. 2000;27:2198-202.

26. Chung YM, Liao H-T, Lin Y-C, et al. Prevalence of spondylarthritis in 504 Chinese patients with HLA-B27-associated acute anterior uveitis. Scand J Rheumatol. 2009;38:84-90.

27. Fernández-Melón J, Munoz-Fernandez S, Hidalgo V, et al. Uveitis as the initial clinical manifestation in patienst with spondylarthropathies. J Rheumatol. 2004;31:524-527.

28. Haroon M, O'Rourke M, Ramasamy P, Murphy CC, FitzGerald O. A novel evidence-based detection of undiagnosed spondyloarthritis in patients presenting with acute anterior uveitis: the DUET (Dublin Uveitis Evaluation Tool). Ann Rheum Dis. 2015;74(11):1990-5.

29. Rudwaleit M, Rodevand E, Holck $P$ et al. Adalimumab effectively reduces the rate of anterior uveitis flares in patients with active ankylosing spondylitis: results of a prospective open-label study. Ann Rheum Dis. 2008:68:696-701.

30. The incidence of extra-articular manifestations in participants with ankylosing spondylitis treated with Golimumab (MK-8259-012) (GOEASY) ClinicalTrials.gov Identifier: NCT01668004.

31. van der Horst-Bruinsma I, van Bentum R, Verbraak FD, et al. The impact of certolizumab pegol treatment on the incidence of anterior uveitis flares in patients with axial spondyloarthritis: 48-week interim results from C-VIEW. RMD Open. 2020;6:e001161. 